\title{
Incidence, aetiology and clinical features of eosinophilic pleural effusion: a retrospective study
}

Minfang $\mathrm{Li}^{1,2 \dagger}$, Yunxiang Zeng ${ }^{3 \dagger}$, Yaqing $\mathrm{Li}^{1}$, Dan Jia ${ }^{1}$, Sheng Chen ${ }^{1 * \dagger}$ and Jinlin Wang ${ }^{3^{*}+}$

\begin{abstract}
Background: Eosinophilic pleural effusion (EPE) is a distinct entity among pleural effusions, but its diagnostic and prognostic significance is still controversial. This study aimed to evaluate the incidence and aetiological distribution of EPE in our institution and to assess the relationship between EPE and malignancy and other underlying diseases and the relevance of the percentage of eosinophils and other laboratory parameters.
\end{abstract}

Methods: A retrospective study was conducted by reviewing the medical records of 252 patients with PE from September 2017 to January 2021.

Results: EPE was found in 34 (13.49\%) out of 252 patients. There were 20 (58.82\%) males and 14 (41.18\%) females in the EPE group. The mean percentage of eosinophils in EPE (21.7\%, range (10.0-67.5\%)) was significantly higher than the percentage of eosinophils in peripheral blood (5.65\%, range (0-34.60\%); $p<0.05)$. The most common cause of EPE was malignant disease (52.94\%), followed by idiopathy (14.71\%), parasites (8.82\%), pneumonia (8.82\%) and others (14.71\%). Comparative analysis of patients with malignant versus nonmalignant EPE showed that patients with malignant EPE were significantly older, and had a lower white blood cell (WBC) count in the pleural fluid (1.8 vs 4.7 cells $\left.\times 10^{9} / \mathrm{L}, p<0.05\right)$. However, the percentage of eosinophils in PE was not significantly different between malignant EPE and nonmalignant EPE $(p=0.66)$. There was no correlation between the percentage of eosinophils in PE and peripheral blood $(r=0.29 ; p=0.09)$.

Conclusions: Malignant disease ranks as the leading cause of EPE. The presence of EPE should not be considered as a predictive factor of benign conditions. Pleural parasitic infestation (PPI) should be emphasized in areas with a high incidence of parasitic disease.

Keywords: Eosinophilic pleural effusion, Eosinophils, Pleural effusion, Pleural fluid, Pleural fluid eosinophilia

\footnotetext{
*Correspondence: chenshengzyy@126.com; drjlwang@126.com

${ }^{\dagger}$ Minfang Li, Yunxiang Zeng, Sheng Chen, and Jinlin Wang have contributed equally to this work

1 Department of Respiratory Medicine, Shenzhen Traditional Chinese Medicine Hospital, Shenzhen 518033, Guangdong Province, China ${ }^{3}$ Department of Respiratory Disease, The State Key Laboratory of Respiratory Disease, China Clinical Research Centre for Respiratory Disease, Guangzhou Institute of Respiratory Health, First Affiliated Hospital of Guangzhou Medical University, 151 Yanjiang Road, Guangzhou 510120, Guangdong Province, China

Full list of author information is available at the end of the article
}

\section{Background}

Eosinophilic pleural effusion (EPE) was first described by Harmsen in 1894 [1]. Since then, it has been of interest to clinicians. EPE, defined as a pleural effusion (PE) in which eosinophils comprise $\geq 10 \%$ of white blood cells (WBCs) [2], accounts for $5-16 \%$ of exudative pleural effusions [2,3].

The diagnostic value and prognostic significance of EPE are still a matter of debate, as it can be a manifestation of a great variety of diseases including infections 
(bacteria, fungi, mycobacteria, parasites), malignancies, autoimmune diseases, drug reactions, pulmonary embolism, chest trauma, asbestos exposure and many others [2-5]. Early studies reported that air/blood was the most common cause of EPE (29\%), and it was once believed that the finding of pleural fluid eosinophilia in an exudative effusion considerably reduced the probability of malignancy and conversely increased the likelihood of an underlying benign disorder [2-5]. However, current studies, performed in the last 2 decades, have confirmed that malignancy accounts for $22.7-40.1 \%$ of EPEs and is the most common aetiology of EPEs [4-9]. This disparity could probably be explained by different study populations, and the various disease spectra of EPEs were a reflection of the populations studied [5].

Due to the rarity of EPE, our knowledge of this phenomenon is based on small series and case reports until now. We are aware of no related report in China. We performed an analysis of a series of samples of PE in our institution. This study aimed to investigate the incidence, aetiology and epidemic characteristics of EPE in our institution.

\section{Methods}

\section{Study design and setting}

A retrospective study of patients with PE was performed at a dedicated respiratory centre (State Key Laboratory of Respiratory Disease and China Clinical Research Centre of Respiratory Disease, Guangzhou Institute of Respiratory Disease, Guangzhou) between September 2017 and January 2021. The study design and protocol were approved by the Ethics Committee of the First Affiliated Hospital of Guangzhou Medical University, and the study was conducted in accordance with the 1964 Declaration of Helsinki and its later amendments. Approval for a waiver of informed consent for the study was obtained from the Institutional Review Board of Guangzhou Medical University.

\section{Patients}

The available data for a total of 252 patients with PE were reviewed retrospectively.

The inclusion criteria for patients with EPE were pleural fluid containing $\geq 10 \%$ of eosinophils.

The inclusion criteria for patients with tuberculous pleural effusion (TPE) were as follows: (1) chronic granulomatous inflammation in pleural tissue; (2) a clinical response to anti-tuberculosis treatment; and (3) no pleural effusion or only a small amount observed in chest ultrasound examinations over a 12-month follow up period.

The criteria of MPE (malignant pleural effusion) were: (1) a positive pleural fluid cytology and/or positive histology of pleural biopsy (proven malignant effusion); or (2) a known malignant disease, after the exclusion of alternative causes of PE (probable malignant effusion) [7].

The criteria for inclusion of patients with PPE (parapneumonic effusion) were as follows: (1) exudative effusions associated with bacterial pneumonia, lung abscesses, or bronchiectasis; (2) absence of Mycobacterium tuberculosis (MTB) in pleural fluid obtained from serial thoracentesis procedures; (3) pathological manifestations of inflammatory pleuritis, pleural fibrosis and plaques, or chronic empyema, without evidence of MTB; and (4) remission and recovery for at least 3 months at follow-up visits after antibiotic treatment.

The inclusion criteria for enrolment of patients with PPI (pleural parasitic infestation): (1) parasite exposure; (2) immunoserologic test result for a parasite-specific antibody, and/or on the detection of characteristic parasite eggs (in the pleural effusion, sputum, bronchial washing fluid, lung biopsy specimens or stool); (3) patients with presumptive diagnosis of PPIs who were responsive to antiparasitic treatment and were followed up for up to 15 months.

CTDs (Connective tissue diseases) were diagnosed in patients with a known specific CTD after the exclusion of other causes of PE.

HRPE (heart related pleural effusion) was identified in patients with definite heart disease followed by exclusion of other causes of PE.

The aetiology of PE was established based on the medical history, physical examination, imaging studies, laboratory findings and pleural fluid and pleural biopsy examination results. Almost all cases of idiopathic eosinophilic pleural effusions (IEPE) had sufficient follow-up periods to exclude malignancy or tuberculosis (more than 1 year).

\section{Statistical analysis}

Continuous variables are presented as the median and range or the mean and standard deviation, and qualitative variables are presented as the number and percentage. Intergroup differences were analysed statistically using SPSS ${ }^{\circledR} 19.0$ (SPSS Inc., Chicago, IL, USA). The Mann-Whitney test or Chi-square test was used for comparisons of the test results. Significance for statistical analyses was set at $p<0.05$.

\section{Results}

\section{Patient characteristics}

EPE was found in 34 (13.49\%) out of 252 patients. There were $20(58.82 \%)$ males and $14(41.18 \%)$ females in the EPE group, and the male to female ratio of nearly 1.43:1 was lower than that for all patients with PE seen during the study period (1.8:1). The mean age of patients in the 
EPE group was $56.41 \pm 15.3$ years (range $25-88$ yreas). Their clinical characteristics are shown in Table 1 . The symptoms presented by the patients included dry cough, shortness of breath, fever, chest pain, and sputum. The duration of complaints ranged from 2 days to more than 2 years. Twenty-seven $(79.41 \%)$ patients had unilateral PE: 16 (59.26\%) left-sided and 11 (40.74\%) rightsided. Bilateral PEs were found in 5 (14.71\%) patients, and polyserositis occurred in $2(5.88 \%)$ patients. Thirtythree (97.06\%) EPEs were identified as exudates, and one (2.94\%) was identified as a transudate.

\section{Laboratory findings}

The mean percentage of eosinophils in EPE (21.7\%, range (10.0-67.5\%)) was significantly higher than the percentage of eosinophils in peripheral blood $(5.65 \%$, range $(0-34.60 \%) ; p<0.05)$. The mean of eosinophil count in the pleural fluid $\left(942.4(11.8-8682.5)\right.$ cells $\left.\times 10^{6} / \mathrm{L}\right)$ was also higher than that in peripheral blood (514.7 $(0-5778.0)$ cells $\left.\times 10^{6} / \mathrm{L}, p<0.05\right)$. The distribution of eosinophil percentage in patients with EPE is shown in Fig. 1. Patients with less than $20 \%$ eosinophils accounted for $64.7 \%$ of patients with EPE. In $14.7 \%$ of patients with EPE, the percentage of eosinophils was greater than $30 \%$. When eosinophilic and noneosinophilic effusions were compared, there were significant differences in the number of eosinophils in peripheral blood, the amount of $\mathrm{WBCs}$ and the level of adenosine deaminase (ADA) in effusions (Table 2).

\section{Aetiology of EPE}

The aetiological distribution of EPEs is exhibited in Table1. The most common cause of EPE was malignant disease (52.94\%) followed by IEPE (14.71\%), PPI (8.82\%), PPE (8.82\%) and others (14.71\%). Lung adenocarcinoma accounted for more than half of all MEPE (14, 77.78\%). The other malignancies were pancreatic cancer, hepatic epithelioid haemangioendothelioma, lung squamous cell carcinoma, and colorectal cancer. In $14.71 \%$ of patients diagnosed with IEPE, the origin of EPE remained unknown after extensive evaluation, including pleural biopsy. The quantity of their pleural effusion was small and did not relapse after the first thoracentesis. None of these patients had an exposure history to asbestos or drugs known to lead to EPE.

Among the 5 patients with PPIs, 2 had a history of raw fish ingestion or raw freshwater crab ingestion. The other 3 patients did not report an exposure history. IgG antibodies against Paragonimus westermani were found in the serum of 3 patients. Echinococcus granulosus and Taenia solium specific antibodies were detected in the blood samples of 2 other individuals. The diagnosis of PPI was suspected and the patients were treated with antiparasitic agents. All of them recovered, and no recurrences were observed by follow-up.

When eosinophilic and noneosinophilic effusions were compared, the prevalence of PPI and IPE appeared higher in EPE than in non-EPE. The occurrence of other causes, including PPE and MPE, was similar in the two groups (Table 3).

\section{Pleural fluid eosinophilia and malignancy}

Comparative analysis of patients with malignant versus nonmalignant EPE showed that patients with malignant EPE were significantly older and had a smaller WBC count in the pleural fluid $\left(1.8\right.$ vs 4.7 cells $\left.\times 10^{9} / \mathrm{L}, P<0.05\right)$ (Table 4). However, the percentage of eosinophils in PE was not significantly different between malignant EPE and nonmalignant EPE $(p=0.66)$.

\section{Pleural fluid eosinophilia and other laboratory parameters} In EPE, there was a positive correlation between the percentage of eosinophils and ADA in PE $(r=0.383$ $p=0.025)$, and a reverse correlation between eosinophils and lactate dehydrogenase (LDH) in PE $(r=-0.396$, $p=0.021$ ). There was a significant correlation between the percentage of eosinophils in peripheral blood and WBC count in PE $(\mathrm{r}=0.453, p=0.007)$. However, there was no correlation between the percentage of eosinophils in $\mathrm{PE}$ and peripheral blood $(\mathrm{r}=0.29 ; p=0.09)$. There were no noteworthy relationships between eosinophils in PE and other laboratory parameters. There was no significant difference between cases of EPE with peripheral eosinophilia and those with normal peripheral eosinophil count (Table 4).

\section{Discussion}

This study included 252 patients with PE diagnosed at a respiratory health research institution, and 34 patients were confirmed to have EPE. To our knowledge, this study represents the largest single study group in our country. Patients with EPE accounted for 13.49\% (34/252) of all patients with PE. The percentages were similar to those in previous studies, which reported $12.6 \%$ [4] and 10\% [2], but higher than those in two other studies, which indicated 5-8\% [2] and 7.2\% [7]. However, Chu FY found that the morbidity of EPE was only $2.9 \%$ [6]. The discrepancy may be attributed to different populations, epidemic characteristics, test methods or the timing of pleural fluid collection. The age of patients with EPE is similar to that in previous reports. The ratio of males to females among EPE patients was 1.43:1, which was lower than the 2:1 ratio reported in previous literature [7]. The higher occurrence of PE in males than in females may explain the ratio of males to females among EPE patients. 


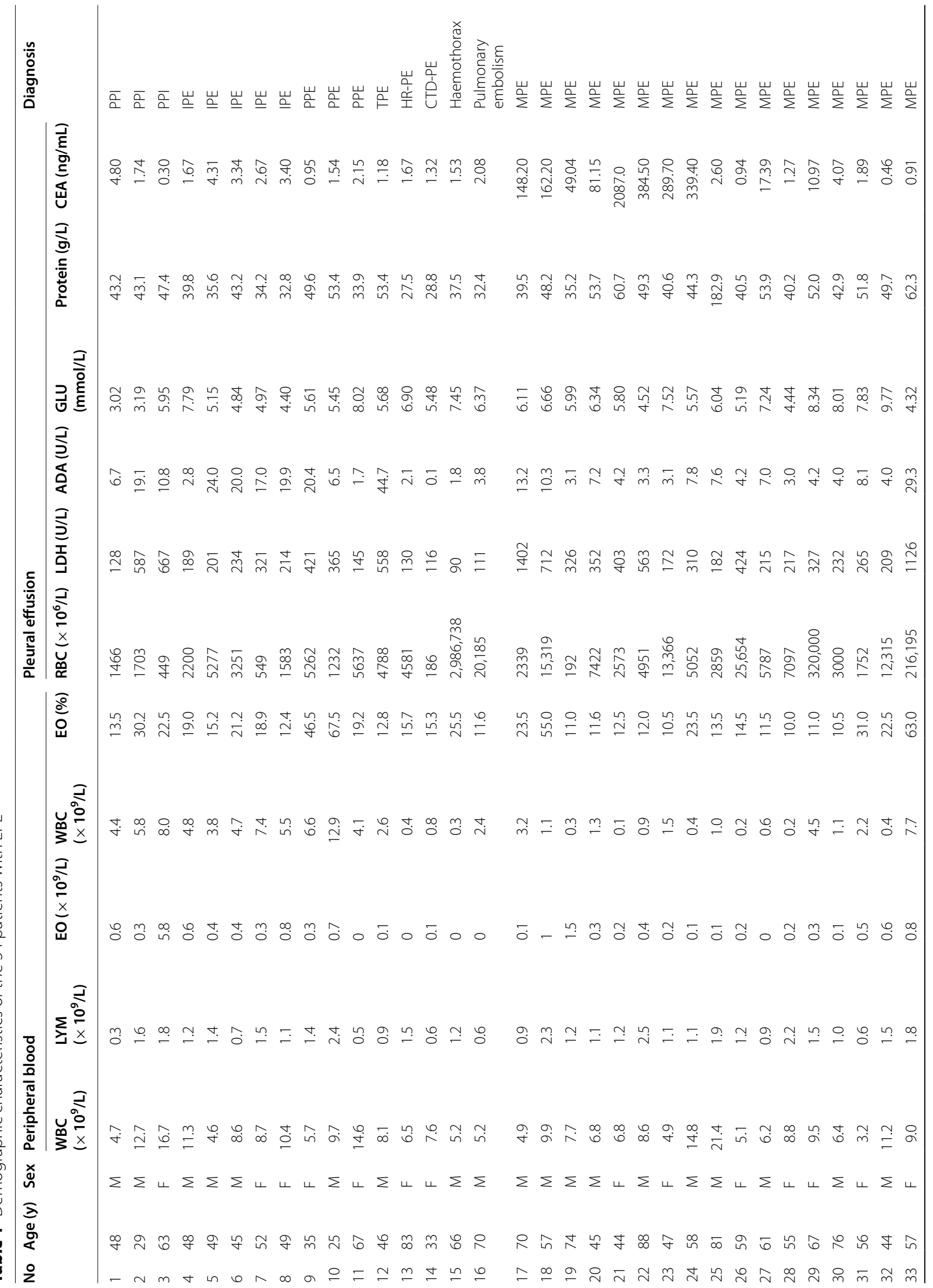




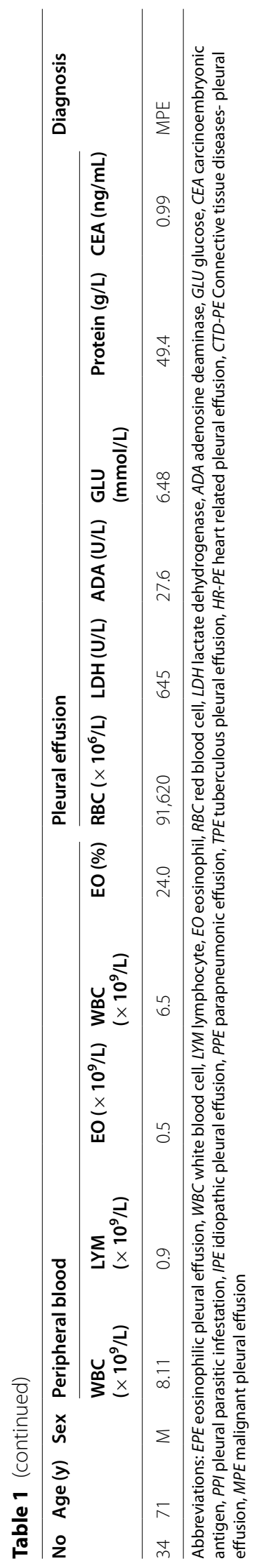




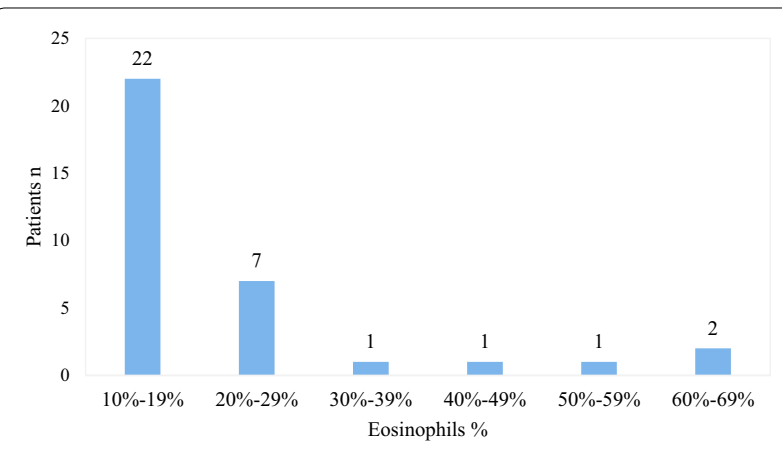

Fig. 1 Distribution of eosinophil percentage in 34 patients with EPE

EPE can be associated with a wide range of underlying conditions, including infections, malignancies, autoimmune diseases, drug reactions, pulmonary embolism, chest trauma and many others [7]. This study indicates that malignant disease was the leading cause of EPE, followed by PPI, IEPE and PPE in sequence. Compared with non-EPE, the prevalence of IPE and PPI was higher in EPE, while TPE was lower. The aetiological distribution of EPE varies among previous reports. In Krenke's study, malignancy (34.8\%), infections (19.3\%), unknown causes $(14.1 \%)$ and posttrauma (8.9\%) were the top four aetiologies in EPE [7]. Oba reported that the most common cause of EPE was malignancy (26\%), followed by idiopathic (25\%) and parapneumonic (13\%) effusions, pleural air/blood (13\%), tuberculosis (7\%), transudate (7\%), other (6\%) and CVD (collagen vascular disease) (3\%) [5]. Wysenbeek's study showed that the aetiologies of EPE were trauma (39\%), congestive heart failure (14\%), infection (8.5\%) and idiopathic effusion (8.5\%) [10]. The differences in results may be explained by the different prevalences of the aetiology of PE at some institutions [11]. Although there was no drug-induced EPE in our study, the list of drugs associated with EPE include cardiology and internal medicine (warfarin, diltiazem, simvastatin and mesalamine), antibiotics (furantoin, daptomycin and tosufloxacin), psychiatric drugs and neurodrugs (valproic acid, dantrolene) [12-14]. Adverse drug reactions should be considered in the differential diagnosis following thorough investigation for other potential causes of EPE.

The correlation between EPE and malignancy is still a subject of debate. Our study indicated that malignant disease, accounting for $52.94 \%$ of EPE cases, was the most common cause associated with EPE. However, it was once believed that the finding of pleural fluid eosinophilia in an exudative effusion considerably reduced the probability of malignancy and conversely increased the likelihood of an underlying benign disorder [15]. Bower and Wysenbeek reported that air/ blood was the most common cause of EPE [10, 15]. However, the spectrum of EPEs has changed since 1960, and malignancy should no longer be considered uncommon among EPEs [5].

Table 2 Comparison of clinical findings of patients with EPE and n-EPE

\begin{tabular}{|c|c|c|c|c|}
\hline & Total $(n=252)$ & $\operatorname{EPE}(n=34)$ & $n-E P E(n=218)$ & $p$-Value \\
\hline Age (y) & $58(19-98)$ & $56.4(25-88)$ & $58.2(19-98)$ & 0.56 \\
\hline Sex & & & & 0.48 \\
\hline Male & $162(64.3 \%)$ & $20(58.8 \%)$ & $142(65.1 \%)$ & \\
\hline Female & $90(35.7 \%)$ & $14(41.2 \%)$ & $76(34.9 \%)$ & \\
\hline \multicolumn{5}{|l|}{ Peripheral blood } \\
\hline WBC $\left(\times 10^{9} / L\right)$ & $8.2(3.0-42.4)$ & $8.6(3.2-21.4)$ & $9.3(3.0-42.4)$ & 0.42 \\
\hline $\operatorname{LYM}\left(\times 10^{9} / \mathrm{L}\right)$ & $1.4(0.2-13.5)$ & $1.3(0.3-2.5)$ & $1.3(0.2-13.5)$ & 0.93 \\
\hline $\mathrm{EO}\left(\times 10^{6} / \mathrm{L}\right)$ & $253.7(0-6200.0)$ & $514.7(0-5778.0)$ & $168.3(0-6200.0)$ & 0.001 \\
\hline \multicolumn{5}{|l|}{ Pleural effusion } \\
\hline $\mathrm{LDH}(\mathrm{U} / \mathrm{L})$ & $943(25-44,144)$ & 369 (90-1402) & $1098(25-44,144.0)$ & 0.30 \\
\hline GLU (mmol/L) & $5.80(0.12-50.21)$ & $6.07(3.02-9.77)$ & $5.70(0.12-50.21)$ & 0.64 \\
\hline Protein (g/L) & $77.1(5.2-182.9)$ & 48.03 (27.5-182.9) & $47.8(5.2-57.3)$ & 0.66 \\
\hline ADA (U/L) & $19.5(0.1-196.7)$ & $10.4(0.1-44.7)$ & $22.4(0.1-196.7)$ & 0.007 \\
\hline CEA (ng/mL) & $715.60(0.2-69,984.0)$ & $106.39(0.30-2087.0)$ & $770.80(0.2-69,984.0)$ & 0.74 \\
\hline WBC $\left(\times 10^{9} / L\right)$ & $1.5(0-12.9)$ & $3.2(0.1-12.9)$ & $1.2(0-8.6)$ & 0.001 \\
\hline EO (\%) & $5.0(0-67.5)$ & $21.7(10.0-67.5)$ & $1.3(0-8.0)$ & $<0.01$ \\
\hline $\mathrm{RBC}\left(\times 10^{9} / \mathrm{L}\right)$ & $44.6(0.08-990,000)$ & $111.2(0.2-2986.7)$ & $30,607.0(0.7-990,000)$ & 0.36 \\
\hline
\end{tabular}

Data are presented as the mean (range) or $\mathrm{n}(\%)$

$E P E$ eosinophilic pleural effusion, WBC white blood cell, $L Y M$ lymphocyte, $E O$ eosinophil, $R B C$ red blood cell, $L D H$ lactate dehydrogenase, $A D A$ adenosine deaminase, GLU glucose, CEA carcinoembryonic antigen 
Table 3 Comparison of aetiology of patients with EPE and n-EPE

\begin{tabular}{llllr}
\hline & Total $(\mathbf{n}=\mathbf{2 5 2})$ & EPE $(\mathbf{n}=\mathbf{3 4})$ & $\mathbf{n}$-EPE $(\mathbf{n}=\mathbf{2 1 8})$ & $\boldsymbol{p}$-Value \\
\hline PPE & $36(14.29 \%)$ & $3(8.82 \%)$ & $33(15.14 \%)$ & 0.475 \\
TPE & $47(18.65 \%)$ & $1(2.94 \%)$ & $46(21.10 \%)$ & 0.011 \\
MPE & $108(42.86 \%)$ & $18(52.94 \%)$ & $90(41.28 \%)$ & 0.201 \\
HR-PE & $34(13.49 \%)$ & $1(2.94 \%)$ & $33(15.14 \%)$ & 0.096 \\
CTD-PE & $7(2.78 \%)$ & $1(2.94 \%)$ & $6(2.75 \%)$ & 0.951 \\
IPE & $6(2.38 \%)$ & $5(14.71 \%)$ & $1(0.46 \%)$ & $<0.001$ \\
PPI & $5(1.98 \%)$ & $3(8.82 \%)$ & $2(0.92 \%)$ & 0.014 \\
Others & $9(3.57 \%)$ & $2(5.88 \%)$ & $7(3.21 \%)$ & 0.776 \\
\hline
\end{tabular}

Data are presented as $\mathrm{n}(\%)$

$E P E$ eosinophilic pleural effusion, $n-E P E$ non-eosinophilic pleural effusion, $P P E$ parapneumonic effusion, TPE tuberculous pleural effusion, MPE malignant pleural effusion, $H R-P E$ heart related pleural effusion, $C T D-P E$ Connective tissue diseases pleural effusion, PPI pleural parasitic infestation, IPE idiopathic pleural effusion

The cumulative incidence of malignancy among EPEs has gradually increased from 7 to $25 \%$ over the last 4 decades [5]. Current studies (studies performed in the last 2 decades) confirmed that malignancy was the leading aetiology of EPE (accounting for 22.7-40.1\% of EPEs) [2, 4, 5, $7,16,17]$. This tendency may be explained by the development of diagnostic technology, improved diagnostic awareness, disparities in the study populations, or varying disease spectra over time [17].

In a recent study, the majority of malignant pleural effusion (MEPE) was associated with lung cancer. According to a literature review, a vast majority of MEPE is related to solid tumours, and only a small group of patients with haematological malignancies develop EPE $[2,3,6,9,16,18-21]$. Lung cancer and metastatic cancer to the lung were the leading causes of MEPE, including solid tumours from other sites and haematological malignancies. The percentage of MEPE with an unknown primary site of cancer accounted for $5-10 \%$ of patients with MEPE [7]. Pathological classification included adenocarcinoma, squamous cell carcinoma, and dysgerminoma $[18,19]$.

It has also been recognized that a high proportion of idiopathic effusions are characterized by EPE [2, 22]. Ferreiro et al. found that the most frequent aetiology of EPE was known, accounting for $36 \%$ of EPE [17]. The percentage of IEPE (14.71\%) was just secondary to MPE in a recent study. It has been reported that the prevalence of IEPE varies from 0 to $67 \%[17,23]$. In the last 2 decades, approximately $3.8-32.1 \%$ of EPE cases were diagnosed as IEPE $[5,19,24]$. Thus, as IEPE appeared to be an important part of EPE with obscure pathogenesis, our previous study advocated that complete medical, surgical and

Table 4 Comparison of clinical findings of patients with MEPE and non-MEPE, peripheral eosinophilia and normal peripheral eosinophil

\begin{tabular}{|c|c|c|c|c|c|c|c|}
\hline & \multirow[t]{2}{*}{ Total $(n=34)$} & \multicolumn{3}{|c|}{ MEPE vs non-MEPE } & \multicolumn{3}{|c|}{ Peripheral eosinophilia vs normal peripheral eosinophil } \\
\hline & & MEPE $(n=18)$ & Non-MEPE $(n=16)$ & $p$-Value & $\begin{array}{l}\text { Peripheral eosinophilia } \\
(\mathrm{n}=9)\end{array}$ & $\begin{array}{l}\text { Normal peripheral } \\
\text { eosinophil }(n=25)\end{array}$ & $p$-Value \\
\hline Age (y) & $56.4(25-88)$ & $61.7(44-88)$ & $50.5(25-83)$ & 0.03 & $51.7(25-74)$ & $58.1(29-88)$ & 0.36 \\
\hline Sex & & & & 0.774 & & & 0.70 \\
\hline Male & $20(58.82 \%)$ & $11(61.11 \%)$ & $9(77.8 \%)$ & & $6(66.7 \%)$ & $14(56.0 \%)$ & \\
\hline Female & $14(41.18 \%)$ & $7(38.89 \%)$ & $7(22.2 \%)$ & & $3(33.3 \%)$ & $11(44.0 \%)$ & \\
\hline \multicolumn{8}{|c|}{ Peripheral blood } \\
\hline WBC $\left(\times 10^{9} / L\right)$ & $8.6(3.2-21.4)$ & $8.5(3.2-21.4)$ & $8.8(4.6-16.7)$ & 0.87 & $10.1(4.7-16.7)$ & $8.1(3.2-21.4)$ & 0.04 \\
\hline $\operatorname{LYM}\left(\times 10^{9} / \mathrm{L}\right)$ & $1.3(0.3-2.5)$ & $1.4(0.6-2.5)$ & $1.2(0.3-2.4)$ & 0.26 & $1.5(0.3-2.4)$ & $1.2(0.5-2.5)$ & 0.13 \\
\hline $\mathrm{EO}\left(\times 10^{6} / \mathrm{L}\right)$ & $514.7(0-5778.0)$ & $394.4(0-1500.0)$ & $661.2(0-5778.0)$ & 0.44 & $1378.0(600-5778.0)$ & $204.0(0-500)$ & $<0.001$ \\
\hline \multicolumn{8}{|c|}{ Pleural effusion } \\
\hline $\mathrm{LDH}(\mathrm{U} / \mathrm{L})$ & $369(90-1402)$ & 449 (172-1402) & $280(90-667)$ & 0.09 & $437(128-1126)$ & $345(90-1402)$ & 0.51 \\
\hline GLU (mmol/L) & $6.07(3.02-9.77)$ & $6.50(4.32-9.77)$ & $5.60(3.02-8.0)$ & 0.12 & $5.93(3.02-9.77)$ & $6.12(3.19-8.34)$ & 0.54 \\
\hline Protein (g/L) & $48.0(27.5-182.9)$ & $55.4(35.2-182.9)$ & $39.7(27.5-53.4)$ & 0.07 & $45.8(32.8-62.3)$ & $48.8(27.5-182.9)$ & 0.73 \\
\hline $\mathrm{ADA}(\mathrm{U} / \mathrm{L})$ & $10.4(0.1-44.7)$ & $8.4(3.0-29.3)$ & $12.6(0.1-44.7)$ & 0.24 & $10.4(2.8-29.3)$ & $10.4(0.1-44.7)$ & 0.79 \\
\hline CEA (ng/mL) & $106.4(0.3-2087.0)$ & $199.0(0.5-2087.0)$ & $2.2(0.3-4.8)$ & 0.11 & $24.9(0.30-162.2)$ & $135.7(1.0-2087.0)$ & 0.32 \\
\hline WBC $\left(\times 10^{9} / \mathrm{L}\right)$ & $3.2(0.1-12.9)$ & $1.8(0.1-7.67)$ & $4.7(0.3-12.9)$ & 0.01 & $5.01(0.3-12.9)$ & $2.50(0.1-7.4)$ & 0.10 \\
\hline EO (\%) & $21.7(10.0-67.5)$ & $20.6(10.0-63.0)$ & $22.9(11.6-67.5)$ & 0.66 & $31.8(11.0-67.5)$ & $18.1(10-46.5)$ & 0.16 \\
\hline $\operatorname{RBC}\left(\times 10^{9} / \mathrm{L}\right)$ & $111.2(0.2-2986.7)$ & $41.0(0.2-320)$ & $190.3(0.2-2986.7)$ & 0.40 & $27.9(0.2-216.2)$ & $141.3(0.2-2986.7)$ & 0.14 \\
\hline
\end{tabular}

Data are presented as the mean (range) or $\mathrm{n}(\%) . M E P E$ malignant eosinophilic pleural effusion, WBC white blood cell, $L Y M$ lymphocyte, EO eosinophil, $R B C$ red blood cell, $L D H$ lactate dehydrogenase, $A D A$ adenosine deaminase, GLU glucose, CEA carcinoembryonic antigen 
drug-related histories should be obtained and thorough work-up and long-term follow-up should be completed to make differential diagnosis [25].

The percentage of PPI (8.82\%) was the third largest group of patients with EPE in our study. Three patients had PPI consisting of of patients with infection of lung fluke Paragonimus westermani, Toxocara spp.. Consumption of raw or undercooked water or food is the main source of parasite infections. Eosinophilia in PPI has been reported in previous studies of EPEs [2630], but most of these are case reports or small series reports. Interestingly, in a study of EPEs from mainland China, PPIs were responsible for $31.3 \%$ of EPE cases [31]. The high incidence may be explained by the high incidence of patients with PPIs and raw food eating habits in certain areas of the country. Most of the patients with PPI had an exposure history [26, 32]. Therefore, one factor is exposure history. Endemicity and local epidemiology should be taken into consideration when exploring the aetiology of EPE.

This was a retrospective study in a single centre, and the number of patients evaluated was small due to the rare prevalence of EPE. Therefore, a larger, multicentre, prospective study is needed to further explore the epidemic characteristics and clinical significance of EPE.

\section{Conclusions}

In conclusion, malignant disease ranks as the leading cause of EPE, followed by IEPE and PPI. The presence of EPE should not be regarded as a predictive factor of benign conditions. PPIs should be emphasized in areas with a high incidence of parasitic disease.

\section{Abbreviations \\ EPE: Eosinophilic pleural effusion; PE: Pleural effusion; n-EPE: Non-eosinophilic pleural effusion; MTB: Mycobacterium tuberculosis; WBC: White blood cell; LYM: Lymphocyte; EO: Eosinophil; RBC: Red blood cell; LDH: Lactate dehydro- genase; ADA: Adenosine deaminase; GLU: Glucose; CEA: Carcinoembryonic antigen; PPI: Pleural parasitic infestation; IPE: Idiopathic pleural effusion; IEPE: Idiopathic eosinophilic pleural effusion; PPE: Parapneumonic effusion; TPE: Tuberculous pleural effusion; HRPE: Heart related pleural effusion; CTD-PE: Connective tissue diseases- pleural effusion; MPE: Malignant pleural effusion; MEPE: Malignant eosinophilic pleural effusion.}

\section{Acknowledgements}

Editorial assistance with the manuscript was provided by Springer Nature Limited.

\section{Authors' contributions}

Study concept and design: JW. Data collection and data management: YZ, YL, DJ. Study design, statistical analysis and study writing: ML. Data review: SC. Critical review/revision of the manuscript and approval of the final version: All authors. All authors read and approved the final manuscript.

\section{Funding}

The study was supported by grants from the Sanming Project of Medicine in Shenzhen (No: SZSM201812063) and Sustainable Development Science and
Technology Project of Shenzhen Science and Technology Innovation Commission (No: KCXFZ20201221173209027). The funding body had no role in the design of the study, data collection, analysis, interpretation of the data, or writing of the manuscript.

\section{Availability of data and materials}

The datasets supporting the conclusions of this article are included within the article and tables. Additional data may be available from the corresponding author upon reasonable request.

\section{Declarations}

\section{Ethics approval and consent to participate}

The study was approved by the Ethics Committee of the First Affiliated Hospital of Guangzhou Medical University. Approval for a waiver of informed consent for the study was obtained from the Institutional Review Board of

Guangzhou Medical University.

\section{Consent for publication}

Not applicable.

\section{Competing interests}

The authors declare that they have no competing interests.

\section{Author details}

${ }^{1}$ Department of Respiratory Medicine, Shenzhen Traditional Chinese Medicine Hospital, Shenzhen 518033, Guangdong Province, China. ${ }^{2}$ The Second School of Clinical Medical Sciences, Guangzhou University of Chinese Medicine, Guangzhou 510120, Guangdong Province, China. ${ }^{3}$ Department of Respiratory Disease, The State Key Laboratory of Respiratory Disease, China Clinical Research Centre for Respiratory Disease, Guangzhou Institute of Respiratory Health, First Affiliated Hospital of Guangzhou Medical University, 151 Yanjiang Road, Guangzhou 510120, Guangdong Province, China.

Received: 13 July 2021 Accepted: 26 November 2021

Published online: 06 December 2021

\section{References}

1. Harmsen W. Inaug Diss der pat. Dorpat. 1894.

2. Adelman M, Albelda SM, Gottlieb J, Haponik EF. Diagnostic utility of pleural fluid eosinophilia. Am J Med. 1984;77(5):915-20.

3. Kuhn M, Fitting JW, Leuenberger P. Probability of malignancy in pleural fluid eosinophilia. Chest. 1989;96(5):992-4.

4. Martinez-Garcia MA, Cases-Viedma E, Cordero-Rodriguez PJ, et al. Diagnostic utility of eosinophils in the pleural fluid. Eur Respir J. 2000;15(1):166-9.

5. Oba Y, Abu-Salah T. The prevalence and diagnostic significance of eosinophilic pleural effusions: a meta-analysis and systematic review. Respiration. 2012;83(3):198-208.

6. Chu FY, Liou CB, Sun JT, et al. Eosinophilia in pleural effusions: a speculative negative predictor for malignancy. Asian Pac J Cancer Prev. 2016;17(3):1411-4.

7. Krenke R, Nasilowski J, Korczynski P, et al. Incidence and aetiology of eosinophilic pleural effusion. Eur Respir J. 2009;34(5):1111-7.

8. Rubins JB, Rubins HB. Etiology and prognostic significance of eosinophilic pleural effusions. A prospective study. Chest. 1996;110(5):1271-4.

9. Ozkara SK, Turan G, Basyigit I. Clinicopathologic significance of eosinophilic pleural effusions in a population with a high prevalence of tuberculosis and cancer. Acta Cytol. 2007;51 (5):773-81.

10. Wysenbeek AJ, Lahav M, Aelion JA, Kaufmann L. Eosinophilic pleural effusion: a review of 36 cases. Respiration. 1985;48(1):73-6.

11. LiWJ, Lin ZD, Wang JL. A narrative review of malignant eosinophilic pleural effusion: incidence, etiology and prognostic significance. Ann Palliat Med. 2021;10(2):2314-22.

12. Krenke R, Light RW. Drug-induced eosinophilic pleural effusion. Eur Respir Rev. 2011;20(122):300-1.

13. Al-Abcha A, Raziq F, Kherallah S, Alratroot A. Mesalamine-induced eosinophilic pleural effusion. BMJ Case Rep. 2020;13(4):e233886. 
14. Zhang G. Valproic acid-induced eosinophilic pleural effusion: a case report and brief review. Ann Clin Psychiatry. 2020;32(4):288-90.

15. Bower G. Eosinophilic pleural effusion. A condition with multiple causes. Am Rev Respir Dis. 1967;95(5):746-51.

16. Kalomenidis I, Light RW. Pathogenesis of the eosinophilic pleural effusions. Curr Opin Pulm Med. 2004;10(4):289-93.

17. Ferreiro L, San JE, Gonzalez-Barcala FJ, et al. Eosinophilic pleural effusion: Incidence, etiology and prognostic significance. Arch Bronconeumol. 2011:47(10):504-9.

18. Reechaipichitkul W, Chuesakoolvanich K. Eosinophilic pleural effusion in adults at Srinagarind Hospital. Southeast Asian J Trop Med Public Health. 2003;34(2):374-8.

19. Matthai SM, Kini U. Diagnostic value of eosinophils in pleural effusion: a prospective study of 26 cases. Diagn Cytopathol. 2003;28(2):96-9.

20. Lipworth BJ, Dhillon DP. A case of eosinophilic pleural effusion complicating multiple myeloma. Scott Med J. 1988;33(3):278.

21. Chang CJ, Cheng JH, Lin MS, Dai YC, Hsiue TR. Eosinophilic pleural effusion as the first presentation of angioimmunoblastic T cell lymphoma. J Formos Med Assoc. 2007;106(2):156-60.

22. Hirsch A, Ruffie P, Nebut M, Bignon J, Chretien J. Pleural effusion: laboratory tests in 300 cases. Thorax. 1979;34(1):106-12.

23. Lakhotia M, Mehta SR, Mathur D, Baid CS, Varma AR. Diagnostic significance of pleural fluid eosinophilia during initial thoracocentesis. Indian $J$ Chest Dis Allied Sci. 1989;31(4):259-64.

24. Kalomenidis I, Light RW. Eosinophilic pleural effusions. Curr Opin Pulm Med. 2003;9(4):254-60.

25. Luo W, Zeng Y, Shen P, He J, Wang J. Diagnostic procedure for idiopathic eosinophilic pleural effusion: a single-center experience. BMC Pulm Med. 2020;20(1):82

26. Wang J, Luo W, Shen P, He J, Zeng Y. Retrospective study of pleural parasitic infestations: a practical diagnostic approach. BMC Infect Dis. 2019;19(1):576.

27. Hooper C, Lee YC, Maskell N. Investigation of a unilateral pleural effusion in adults: British Thoracic Society Pleural Disease Guideline 2010. Thorax. 2010;65(Suppl 2):i4-17.

28. Jacobson ES. A case of secondary echinococcosis diagnosed by cytologic examination of pleural fluid and needle biopsy of pleura. Acta Cytol. 1973;17(1):76-9.

29. Fang L, Wang Y, Gao Q, Yan B, Zhou J. Recurrent eosinophilic pleuritis caused by sparganum infection: a case report and review of the literature. Medicine (Baltimore). 2020;99(22):e20226.

30. Oh Y, Kim JT, Kim MK, et al. Eosinophilic pleuritis due to sparganum: a case report. Korean J Parasitol. 2014;52(5):541-3.

31. Yongjian Z. Clinical analysis of 50 cases of eosinophilic pleurisy. Med Rev. 1995; 1(6):263-4.

32. Jeon K, Koh WJ, Kim H, et al. Clinical features of recently diagnosed pulmonary paragonimiasis in Korea. Chest. 2005;128(3):1423-30.

\section{Publisher's Note}

Springer Nature remains neutral with regard to jurisdictional claims in published maps and institutional affiliations.

Ready to submit your research? Choose BMC and benefit from:

- fast, convenient online submission

- thorough peer review by experienced researchers in your field

- rapid publication on acceptance

- support for research data, including large and complex data types

- gold Open Access which fosters wider collaboration and increased citations

- maximum visibility for your research: over $100 \mathrm{M}$ website views per year

At BMC, research is always in progress.

Learn more biomedcentral.com/submissions 